\title{
Mössbauer Study of Magnetic Texture of Finemet-Type Ribbons
}

\author{
K. Brzózka ${ }^{a, *}$, P. Sovák ${ }^{b}$, B. Górka ${ }^{a}$, T. Szumiata ${ }^{a}$ And M. GaWroński ${ }^{a}$ \\ ${ }^{a}$ Department of Physics, Technical University of Radom, Krasickiego 54, 26-600 Radom, Poland \\ ${ }^{b}$ Institute of Physics, P.J. Šafárik University, Park Angelinum 9, 04154 Košice, Slovakia
}

\begin{abstract}
Magnetic texture of amorphous Finemet-type alloys substituted by Co, Ni, Mn, Ge and V was investigated by means of Mössbauer spectroscopy using various angular configurations. Samples in form of ribbons were manufactured by single-roller melt spinning technique. In most of investigated alloys some preference of alignment of atomic magnetic moments in-plane of sample is stated, except of some alloys substituted by germanium in which slight tendency to out-of plane spin orientation is proved.
\end{abstract}

PACS: 76.80.+y, 75.50.Kj, 81.40.Ef

\section{Introduction}

The subject of the present paper are magnetic materials belonging to an alloy group called Finemet. The first amorphous alloy from this family, of chemical composition $\mathrm{Fe}_{73.5} \mathrm{Nb}_{3} \mathrm{Cu}_{1} \mathrm{~B}_{9} \mathrm{Si}_{13.5}$, was obtained in 1988 [1] by rapid quenching of melt and had the form of a ribbon. It is known from excellent soft magnetic properties [2] and also as a potential precursor of nanocrystalline alloys that can be manufactured through controlled annealing (under vacuum) of the initial amorphous material at suitably chosen temperature. Many attempts of improving the properties of classical Finemet have been done up to now. They consist in searching the optimal chemical composition [3-8] as well as manufacturing conditions [9] and also thermal treatment terms [10-11].

The most popular method of producing the amorphous alloys is melt spinning. In this method a hot melt of chosen composition is spilled on the surface of rotating copper drum. The process can be carried out in air or under protective argon atmosphere. The melt congeals in the form of $20-30 \mu \mathrm{m}$ thick ribbon. The cooling rate that can be achieved in this method is of order $10^{7} \mathrm{~K} / \mathrm{s}$. As seen, the specific manufacturing conditions cause an anisotropy of final product. We can expect that its inplane and out-of plane properties may be different and additionally a conspicuous axis of anisotropy may occur, for instance - along the ribbon.

In this paper, magnetic texture of various Finemetlike amorphous alloys is the main subject of interest and it is investigated by means of transmission Mössbauer spectroscopy (TMS) as well as conversion electron Möss-

* corresponding author; e-mail: k.brzozka@pr.radom.pl bauer spectroscopy (CEMS). All the samples were manufactured using the same experimental system for meltspinning, in similar conditions, therefore differences in their properties can be attributed mainly to their chemical composition.

\section{Theoretical aspects}

It is known that some information about alignment of atomic magnetic moments can be derived from Mössbauer spectra. In the case of unpolarized dipole radiation, relative intensities of lines 2, 5 in a Zeeman sextet are related to $\left\langle\cos ^{2} \psi_{R}\right\rangle-$ mean value of $\cos ^{2} \psi_{R}$, where $\psi_{R}$ signifies the angle between the incident gamma beam and the local magnetic hyperfine field, with the following relations [12]:

$$
\begin{aligned}
& p=I_{2,5} / I_{3,4}, \quad\left\langle\cos ^{2} \psi_{R}\right\rangle=(4-p) /(4+p), \\
& p=4\left(1-\left\langle\cos ^{2} \psi_{R}\right\rangle\right) /\left(1+\left\langle\cos ^{2} \psi_{R}\right\rangle\right) .
\end{aligned}
$$

In the case of circularly polarized gamma radiation, when Mössbauer spectrum is asymmetric in respect of line intensities, the parameter $p$ can be derived from the formula:

$$
p=\left(I_{2}+I_{5}\right) /\left(I_{3}+I_{4}\right) \text {. }
$$

One can increase the amount of information when some Mössbauer experiments in different geometrical arrangement are performed. Let us consider a magnetic material in form of ribbon and let us choose the coordination system shown in Fig. 1. According to the model presented by J-M Greneche et al. [13-14], parameters: $N_{X}, N_{Y}, N_{Z}$ can be introduced, called "populations", in order to describe mean alignment of atomic magnetic moments. They denominate relative numbers of spins directed along axes $O X, O Y, O Z$, respectively, of a hypothetical discrete structure equivalent to that under 
examination, in which identical angular dependence of $\left\langle\cos ^{2} \psi_{R}\right\rangle$ occurs. If the sample shows mirror symmetry towards $0 X$ axis, the parameters can be derived experimentally by Mössbauer spectroscopy. They fulfill the following relations:

$$
\begin{aligned}
& N_{X}+N_{Y}+N_{Z}=1, \\
& \left\langle\cos ^{2} \psi_{R}\right\rangle_{X}=N_{X} \sin ^{2} \theta \cos ^{2} \phi, \\
& \left\langle\cos ^{2} \psi_{R}\right\rangle_{Y}=N_{Y} \sin ^{2} \theta \sin ^{2} \phi, \\
& \left\langle\cos ^{2} \psi_{R}\right\rangle_{Z}=N_{Z} \cos ^{2} \theta, \\
& \left\langle\cos ^{2} \psi_{R}\right\rangle=N_{X} \sin ^{2} \theta \cos ^{2} \phi+N_{Y} \sin ^{2} \theta \sin ^{2} \phi \\
& \quad+N_{Z} \cos ^{2} \theta,
\end{aligned}
$$

where $\left\langle\cos ^{2} \psi_{R}\right\rangle_{i}, i=X, Y, Z$ are contributions coming from axes $O X, O Y, O Z$, respectively. In other words, we replace the real system by a hypothetical one, in which magnetic moments can lie only along the axes $O X, O Y$, $O Z$ and which gives the same Mössbauer spectrum as the real system.

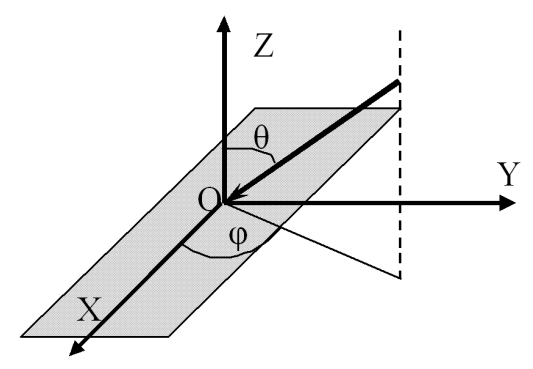

Fig. 1. The coordinations system; $X O Y$ is the sample plane, $O X$ lies along the ribbon.

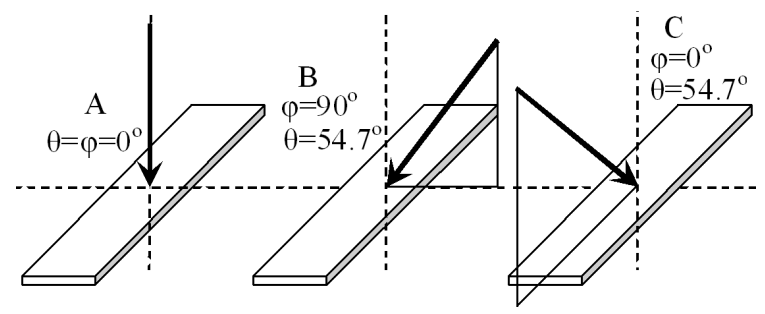

Fig. 2. Configurations of TMS measurements used for texture determination - axes as in Fig. 1, the arrow represents the incident gamma radiation.

The individual populations can be determined from Mössbauer measurements carried out in configurations $A, B, C$ that are defined in Fig. 2. The arrow in the figure represents the incident gamma radiation while coordination axes are similar to those in previous figure. Configuration $A$, in which the gamma radiation reaches perpendicularly the sample surface, is the most popular one. Configurations $B$ and $C$ use the angle $\phi=0$ and $\phi=90^{\circ}$, respectively, and the "magic" angle $\theta \approx 54.7^{\circ}$ that fulfill the relation:

$$
\cos ^{2} \theta=\frac{1}{3},
$$

where $1 / 3$ is an average value of $\cos ^{2} \theta$ for random angular distribution. From relations $(4)-(7)$ one can obtain populations:

$$
\begin{aligned}
N_{Z} & =\left\langle\cos ^{2} \psi_{R}\right\rangle_{A}, \\
N_{Y} & =\frac{1}{2}\left(1-\left\langle\cos ^{2} \psi_{R}\right\rangle_{A}\right) \\
+ & \frac{3}{4}\left(\left\langle\cos ^{2} \psi_{R}\right\rangle_{B}-\left\langle\cos ^{2} \psi_{R}\right\rangle_{C}\right), \\
N_{X} & =\frac{1}{2}\left(1-\left\langle\cos ^{2} \psi_{R}\right\rangle_{A}\right) \\
& -\frac{3}{4}\left(\left\langle\cos ^{2} \psi_{R}\right\rangle_{B}-\left\langle\cos ^{2} \psi_{R}\right\rangle_{C}\right),
\end{aligned}
$$

where $\left\langle\cos ^{2} \psi_{R}\right\rangle_{K}, K=A, B, C$ denote the values obtained from the measurement performed in corresponding configurations.

\section{Experimental details}

Finemet-type alloys in which several atoms of iron or silicon were replaced by atoms of other elements: $\mathrm{Co}, \mathrm{Ni}$, $\mathrm{Mn}, \mathrm{V}$ and Ge, were prepared by single-roller melt spinning technique. Transmission Mössbauer measurements were carried out by use of a conventional spectrometer arranged in vertical geometry with ${ }^{57} \mathrm{Co}(\mathrm{Rh})$ source and a vibrator working in a constant acceleration mode. On the basis of investigations performed using standard configuration $A$, the population of magnetic moments lying in $X O Y$ plane: $N_{X}+N_{Y}=1-N_{Z}$ was derived. For selected alloys the measurements were carried out also in configurations $B$ and $C$ by means of a specially projected sample holder which ensured its proper inclination. It enabled the evaluation of $N_{X}$ and $N_{Y}$ using relations (11), (12). In order to compare the magnetic texture in bulk material and on the surface, conversion electron Mössbauer spectroscopy (CEMS) measurements were performed, using a gas-flow CEMS detector supplied with $\mathrm{He}+4 \% \mathrm{CH}_{4}$ mixture.

\section{Results}

Mössbauer spectra of investigated alloys take a shape typical of the magnetic amorphous materials — a smeared Zeeman sextet, the line width of which increases with the distance of line from the centre of spectrum. The shape of spectrum indicates that it originates from atoms being subjected to distributed magnetic hyperfine fields (MHF). The spectra were fitted by means of programs NORMOS or MOSFIT [15] using histogram-like form of MHF distribution. In both procedures, a linear correlation between magnetic hyperfine field and isomer shift were assumed. Exemplary Mössbauer spectra of $\mathrm{Fe}_{73.5} \mathrm{Cu}_{1} \mathrm{Nb}_{3} \mathrm{Ge}_{x} \mathrm{Si}_{13.5-x} \mathrm{~B}_{9}$ alloys are shown in Figs. 3a$\mathrm{d}$, the values of $p$ are also noticed. 


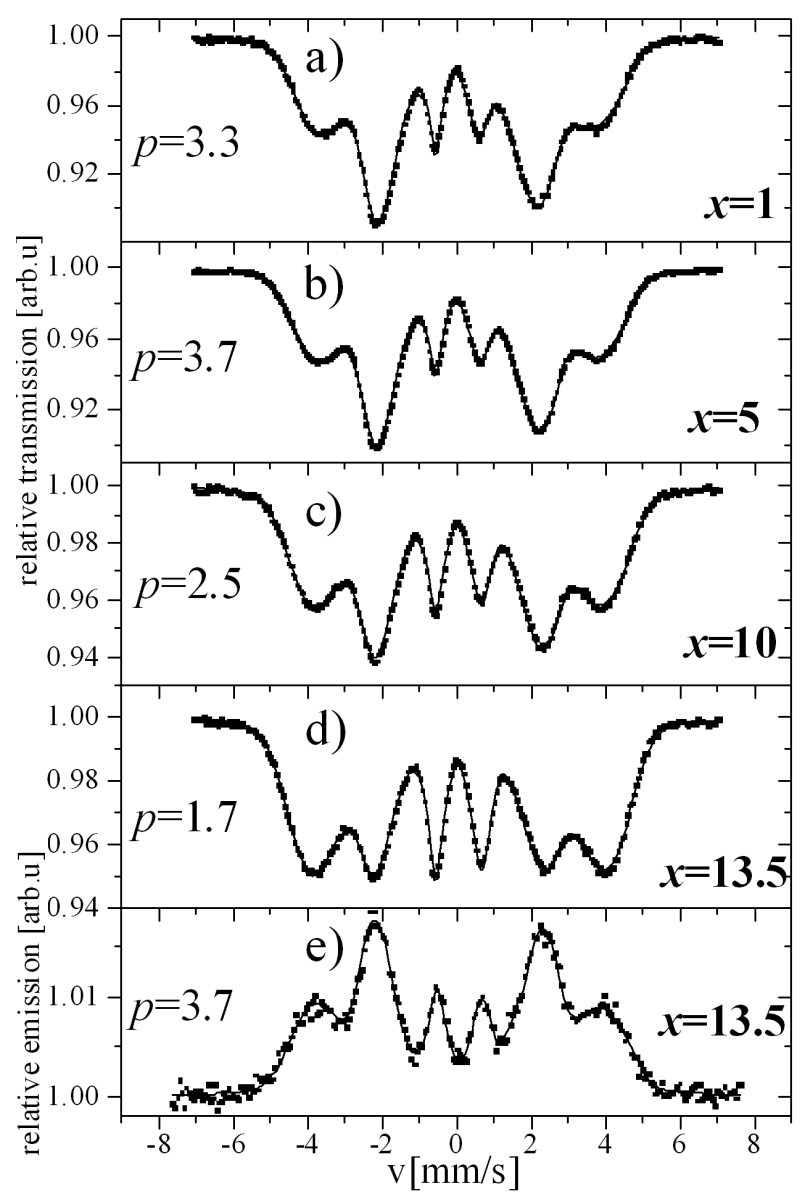

Fig. 3. a-d) TMS spectra of amorphous $\mathrm{Fe}_{73.5} \mathrm{Cu}_{1} \mathrm{Nb}_{3} \mathrm{Ge}_{x} \mathrm{Si}_{13.5-x} \mathrm{~B}_{9} ; \quad$ e) CEMS spectrum of amorphous $\mathrm{Fe}_{73.5} \mathrm{Cu}_{1} \mathrm{Nb}_{3} \mathrm{Ge}_{13.5} \mathrm{~B}_{9}$.

Analogous measurements were carried out for alloys of composition: $\mathrm{Fe}_{73.5-x} \mathrm{Mn}_{x} \mathrm{Cu}_{1} \mathrm{Nb}_{3} \mathrm{Si1}_{3.5} \mathrm{~B}_{9}$, $\mathrm{Fe}_{73.5-x} \mathrm{Ni}_{x} \mathrm{Cu}_{1} \mathrm{Nb}_{3} \mathrm{Si}_{13.5} \mathrm{~B}_{9}, \mathrm{Fe}_{73.5-x} \mathrm{Co}_{x} \mathrm{Cu}_{1} \mathrm{Nb}_{3} \mathrm{Si}_{13.5} \mathrm{~B}_{9}$, $\mathrm{Fe}_{73.5-x} \mathrm{~V}_{x} \mathrm{Cu}_{1} \mathrm{Nb}_{3} \mathrm{Si}_{13.5} \mathrm{~B}_{9}$. In all cases the quantity $p$ was determined and afterwards the values of $N_{X}+N_{Y}$ were calculated. The results are presented in Fig. 4. As seen, in most investigated ribbons parameter $p$ belongs to the range $2.4<p<3.5$ which gives $0.75<N_{X}+N_{Y}<0.93$, while the value characteristic of the random spin orientation equals 0.67 . This means that alignment of magnetic moments in-plane of ribbon is preferred in most alloys. One exception is Finemet substituted with germanium, in which systematic growth of population of magnetic moments oriented out-of plane is observed when increasing $\mathrm{Ge}$ concentration. For $x=13.5$ at $\%$ the parameter $N_{X}+N_{Y}$ equals 0.60 and therefore some slight preference of out-of plane orientation occurs. On average, Finemet alloys substituted by Co show the most distinct tendency for in-plane alignment of spins.

In order to check homogeneity of spin orientation in the $\mathrm{Fe}_{73.5} \mathrm{Cu}_{1} \mathrm{Nb}_{3} \mathrm{Ge}_{13.5} \mathrm{~B}_{9}$ sample, TMS measurement was

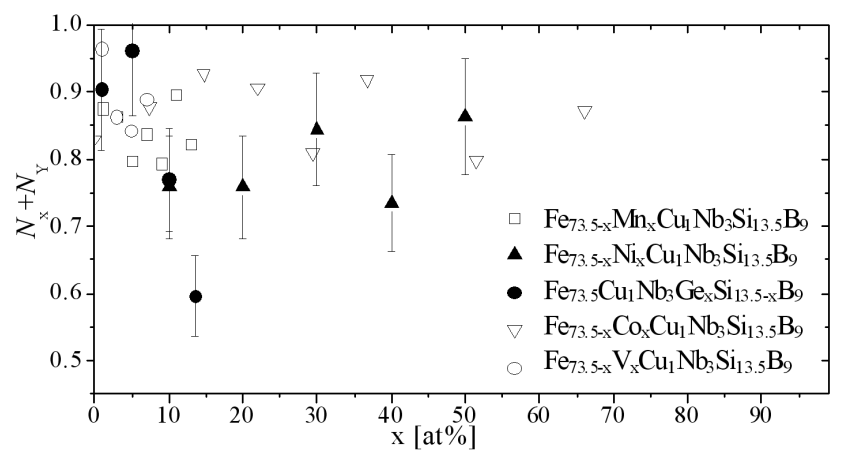

Fig. 4. The population of in-plane oriented spins as a function of added element concentration. For some points, uncertainties of the population are shown.

repeated for other piece of the same ribbon and we obtained the value $p=2.1$ which gives $N_{X}+N_{Y}=0.69$, the value very close to that characteristic of random spin orientation. Since the alignment of magnetic moments in Finemet alloys substituted by Ge seems to be strongly dependent on Ge concentration, we attribute the difference to chemical content fluctuations. CEMS measurement of the former sample was also performed in order to investigate the spin orientation at the surface (Fig. 3e). It was found that $p=3.7$, which is related to $N_{X}+N_{Y}=0.95$. This means nearly full in-plane alignment of atomic magnetic moments at the surface of the ribbon.

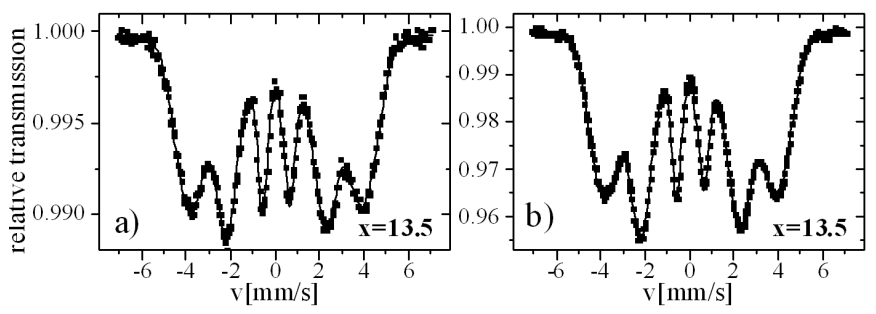

Fig. 5. Mössbauer spectra of $\mathrm{Fe}_{73.5} \mathrm{Cu}_{1} \mathrm{Nb}_{3} \mathrm{Ge}_{13.5} \mathrm{~B}_{9}$ alloy collected using configurations $B$ and $C$.

TABLE

Populations of magnetic moments derived from TMS.

\begin{tabular}{l|l|l|l|l}
\hline \hline Alloy & $N_{X}$ & $N_{Y}$ & $N_{Z}$ & $N_{X}+N_{Y}$ \\
\hline $\mathrm{Fe}_{73.5} \mathrm{Cu}_{1} \mathrm{Nb}_{3} \mathrm{Ge}_{13.5} \mathrm{~B}_{9}$ & 0.433 & 0.163 & 0.404 & 0.596 \\
\hline $\mathrm{Fe}_{51.5} \mathrm{Co}_{22} \mathrm{Cu}_{1} \mathrm{Nb}_{3} \mathrm{Si}_{13.5} \mathrm{~B}_{9}$ & 0.450 & 0.346 & 0.204 & 0.796
\end{tabular}

The alloys of composition $\mathrm{Fe}_{73.5} \mathrm{Cu}_{1} \mathrm{Nb}_{3} \mathrm{Ge}_{13.5} \mathrm{~B}_{9}$ and $\mathrm{Fe}_{51.5} \mathrm{Co}_{22} \mathrm{Cu}_{1} \mathrm{Nb}_{3} \mathrm{Si}_{13.5} \mathrm{~B}_{9}$ were chosen for subsequent TMS measurements using configurations $B, C$. Mössbauer spectra of the former ribbon are shown in Fig. 5 . The determined populations $N_{X}, N_{Y}, N_{Z}$ are presented in Table. It is worth reminding that for fully random orientation of magnetic moments each of the populations equals $1 / 3$. The outcomes prove distinct preference of the 
direction along the ribbon in both samples. Besides, the axis perpendicular to the surface of ribbon is preferred in $\mathrm{Fe}_{73.5} \mathrm{Cu}_{1} \mathrm{Nb}_{3} \mathrm{Ge}_{13.5} \mathrm{~B}_{9}$ alloy while in-plane, transverse direction is favored in $\mathrm{Fe}_{51.5} \mathrm{Co}_{22} \mathrm{Cu}_{1} \mathrm{Nb}_{3} \mathrm{Si}_{13.5} \mathrm{~B}_{9}$.

\section{Summary}

TMS studies prove that in most of the Finemet-type ribbons the population of spin oriented parallel to the ribbon surface fulfills the condition: $0.75<N_{X}+N_{Y}<0.93$ and therefore atomic magnetic moments show strong preference to in-plane alignment. The alloys substituted with germanium seem to be the exception, since the observed tendency diminishes with growing germanium concentration and for 13.5 at\% of Ge the ribbon exhibits nearly random orientation or slight preference to out-of plane spin alignment. As shown from CEMS investigations, even for this alloy nearly full in-plane alignment of atomic magnetic moments is observed at the surface of ribbon. The investigated alloys exhibit also the preferred anisotropy axis along the ribbon.

\section{References}

[1] Y. Yoshizawa, S. Oguma, K. Yamauchi, J. Appl. Phys. 64, 6044 (1988).

[2] G. Herzer, Phys. Scr. 49, 307 (1993).
[3] V.J. Cremaschi, A. Saad, M.J. Ramos, H. Sirkin, J. Alloys Compd. 369, 101 (2004).

[4] S. Atalay, H.I. Adiguzel, P.T. Squire, P. Sovák, Mater. Sci. Eng., A 304-306, 918 (2001).

[5] T. Szumiata, B. Górka, A. Zorkovská, P. Sovák, J. Magn. Magn. Mater. 295, 37 (2005).

[6] P. Sovák, A. Zorkovská, Structure and magnetic properties of Finemet based alloys, P.J. Šafárik University, Košice 2008.

[7] K. Brzózka, M. Gawroński, T. Szumiata, B. Górka, P. Sovák, G. Pavlik, Hyperfine Interact. 183, 235 (2008).

[8] F. Shahri, A. Beitollahi, J. Non-Cryst. Solids 354, 1487 (2008)

[9] S.N. Kane, S. Sarabhai, A. Gupta, L.K. Varga, T. Kulik, J. Magn. Magn. Mater. 215-216, 372 (2000).

[10] C. Miguel, A. Zhukov, J.J. del Val, J. Gonzalez, J. Magn. Magn. Mater. 294, 245 (2005).

[11] T. Kulik, J. Ferenc, M. Kowalczyk, J. Mater. Process. Technol. 162-163, 215 (2005).

[12] H. Frauenfelder, D.E. Nagle, R.D. Taylor, D.R.F. Cochran, W.M. Visscher, Phys. Rev. 126, 1065 (1962).

[13] J.M. Greneche, M. Henry, F. Varret, J. Magn. Magn. Mater. 26, 153 (1982).

[14] J.M. Greneche, F. Varret, J. Phys. 43, L-233 (1982).

[15] J. Teillet, F. Varret, MOSFIT program, Le Mans, unpublished. 